\title{
A simple synthesis of 4-aroyl-5-methyl-1H-imidazol-2(3H)-one derivatives (Enoxymone analogues) from aryl methyl ketones via enaminones
}

\author{
Jure Bezenšek, Uroš Grošelj, Katarina Stare, Jurij Svete, and Branko Stanovnik* \\ Faculty of Chemistry and Chemical Technology, University of Ljubljana Aškerčeva 5, P. O. Box \\ 537, 1000 Ljubljana \\ E-mail: Branko.Stanovnik@fkkt.uni-lj.si
}

\section{Dedicated to Professor Rosa M. Claramunt on the occasion of her $65^{\text {th }}$ anniversary}

\begin{abstract}
Aryl methyl ketones 1a-e gave with $N, N$-dimethylacetamide dimethylacetal (DMADMA) (E)-1aryl-3-(dimethylamino)-but-2-en-1-ones 2a-e. Substitution of the $N, N$-(dimethylamino) group in the reaction with ammonium acetate afforded the corresponding (Z)-3-amino-1-aryl-but-2-en-1ones 3a-e. In the reaction of 3a-e with diethyl azodicarboxylate intermediates 4a-e were formed, which were, in most cases without isolation, cyclized into ethyl (5-aroyl-4-methyl-2-oxo-2,3dihydro-1 $H$-imidazol-1-yl)carbamates 5a-e. Hydrolysis of the ester group, followed by the decarboxylation and deamination of intermediates 6a-c,e produced 4-aroyl-5-methyl-1Himidazol-2(3H)-ones 7a-c,e.
\end{abstract}

Keywords: Aryl methyl ketones, enaminoines, 3-amino-1-arylbut-2-en-1-ones, (2-oxo-2,3dihydro-1H-imidazol-1-yl)carbamates, $1 H$-imidazol-2( $3 H)$-ones.

\section{Introduction}

Nitrogen containing heterocycles ${ }^{1}$ are of special interest in organic synthetic chemistry, since they occur in wide variety of natural products. The imidazolone motif appears in many natural products, ${ }^{2}$ which possess interesting biological activities. ${ }^{3}$ They are inhibitors of V-RAF murine sarcoma viral oncogene homologue B1. ${ }^{4}$ They are antagonists of many receptors including the neurokinin- 1 receptor $^{5}$ and the dopamine receptor. ${ }^{6}$ They were applied as intermediates in the synthesis of many natural products, such as biotin, ${ }^{7}$ slagenins, ${ }^{8}$ axinohydantoins, ${ }^{9}$ oroidin-derived alkaloids, ${ }^{10}$ aplysinopsins, ${ }^{11}$ Lancetta-derived alkaloid carcaridine $\mathrm{A},{ }^{12}$ and others. Due to their importance many methods have been developed for construction of imidazole ring. ${ }^{13,14}$ Recently, there has been a great progress in copper-catalyzed $\mathrm{N}$-arylation. ${ }^{15,16} 4$-Aroyl-1,3-dihydro- $2 \mathrm{H}$ - 
imidazol-2-ones, have been prepared by acylation of the appropriate $2 \mathrm{H}$-imidazol-2-ones and evaluated as a new class of cardiotonic agents. ${ }^{17}$ The most important compound in this series is 5-methyl-4-[4-(methylthio)benzoyl]-1H-imidazol-2(3H)-one (Perfan or Enoximone) (Figure 1), a selective phosphodiesterase inhibitor, has a significant inotropic and vasodilating properties that have proved useful in the postoperative management of infants and children having cardiac surgery. ${ }^{18,19}$ Effects of phosphodiesterase (III/IV)-inhibitors and cytokines on mechanical properties of neutrophilic granulocytes in neonates and adults have been studied. ${ }^{20}$

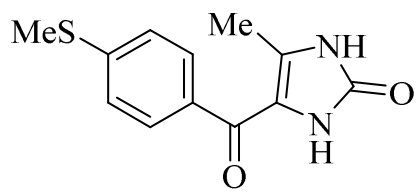

Figure 1. 5-Methyl-4-[4-(methylthio)benzoyl]-1H-imidazol-2(3H)-one (Perfan or Enoximone).

The wide applicability of 3-(dimethylamino)-propenoates and related enaminones as versatile reagents in heterocyclic synthesis, ${ }^{21}$ parallel solution-phase and solid-phase synthesis of fused pyrimidinones, ${ }^{22}$ and stereochemical synthesis, ${ }^{23}$ including natural products and their analogues, e.g. the aplysinopsins, ${ }^{24}$ meridianines, ${ }^{25}$ dipodazines, ${ }^{26}$ and triprostatines ${ }^{27}$ has been demonstrated. Recently, a simple one-pot synthesis of ethyl 4-benzoyl-2-oxo-3-substituted-2,3dihydro- $1 H$-imidazol-1-yl)carbamates has been described. ${ }^{28}$

In this communication we report a simple synthesis of ethyl (5-aroyl-4-methyl-2-oxo-2,3dihydro-1H-imidazol-1-yl)carbamate followed by hydrolysis of the ester group, decarboxylation and deamination to give 4-aroyl-5-methyl-1H-imidazol-2(3H)-ones.

\section{Results and Discussion}

In this paper we report the preparation of 5-methyl-4-(aroyl substituted)imidazol-2(1H)-ones starting from aryl methyl ketones 1a-e, which were transformed by treatment with $N, N$-dimethyl acetamide dimethylacetal (DMADMA) into (E)-3-(dimethylamino)-1-(4-substituted-phenyl)but2-en-1-ones 2a-e. These were treated with ammonium acetate to form (Z)-3-amino-1-(4substituted-phenyl)but-2-en-1-ones 3a-e. By further reaction with diethyl azodicarboxylate (DEAD) intermediates 4a-e were formed which cyclize after addition of sodium hydroxide under experimental conditions into ethyl [5-aroyl-4-methyl-2-oxo-2,3-dihydro- $1 H$-imidazol-1yl]carbamates 5a-e. After hydrolysis of the ester group, followed by decarboxylation and deamination, 4-aroyl-5-methyl-1H-imidazol-2(3H)-ones 7a-c,e were isolated.

In our first attempt to synthesize 5-methyl-4-[(4-methylthio)benzoyl]-1H-imidazol-2(1H)one (7a) (Enoximone or Perfan) we treated 1-[(4-methylthio)phenyl]ethanone (1a) with dimethylacetamide dimethylacetal (DMADMA) in dry toluene to afford (E)-3(dimethylamino)- 
[(4-methylthio)phenyl]but-2-en-1-one (2a) as an yellow oil in $5 \%$ yield. By further treatment of this compound with ammonium acetate in $\mathrm{MeOH}$ for $18 \mathrm{~h}$ at room temperature the corresponding (Z)-3-amino-1-[(4-methylthio)phenyl]but-2-en-1-one (3a) was obtained practically quantitatively as a yellow solid. In the reaction of 3a with diethyl azodicarboxylate (DEAD) in EtOH for $2 \mathrm{~h}$ at room temperature, without the isolation of the intermediate $\mathbf{4 a}$, ethyl [4-methyl-5-(4-methylthio)benzoyl]-2-oxo-2,3-dihydro-1H-imidazol-1-yl]carbamate (5a) was isolated in $68 \%$ yield upon addition of $\mathrm{NaOH}$ to $4 \mathbf{a}$. At the end $\mathbf{5 a}$ was hydrolyzed and decarboxylated by heating at reflux temperature in an aqueous ethanolic $\mathrm{KOH}$ solution for $20 \mathrm{~h}$ to afford 1-amino-4-methyl--(4-methylthio)benzoyl)-1H-imidazol-2(3H)-one (6a) which was directly deaminated to 5-methyl-4-(4-(methylthio)benzoyl)- $1 H$-imidazol-2(3H)-one (7a) in $93 \%$ overall yield. However, the overall yield starting from 1a was very low $(<3 \%)$.

In order to avoid the extremely poor yield in the transformation of $1 \mathbf{a}$ into $\mathbf{2 a}$, we extended the reaction to a number of aryl ketones $\mathbf{1 b - f}$ and prepared the corresponding analogues of enoximone 7a. (Scheme 1, Table 1). We found that 1-(4-fluorophenyl)ethanone (1f) could be transformed with DMADMA into (E)-3-(dimethylamino)-1-1(4-fluorophenyl)but-2-en-1-one (2f) in $67 \%$ yield. The substitution of fluorine with MeSNa afforded (E)-3-(dimethylamino)-1-1(4-(methylthio)phenyl)but-2-en-1-one (2a) in $80 \%$ yield. In this way, following the reaction sequence $\mathbf{1 f} \rightarrow \mathbf{2 f} \rightarrow \mathbf{2 a} \rightarrow \mathbf{3 a} \rightarrow \mathbf{4 a} \rightarrow \mathbf{5 a} \rightarrow \mathbf{6 a} \rightarrow \mathbf{7 a}$, the overall yield was $32 \%$ (Scheme 2).
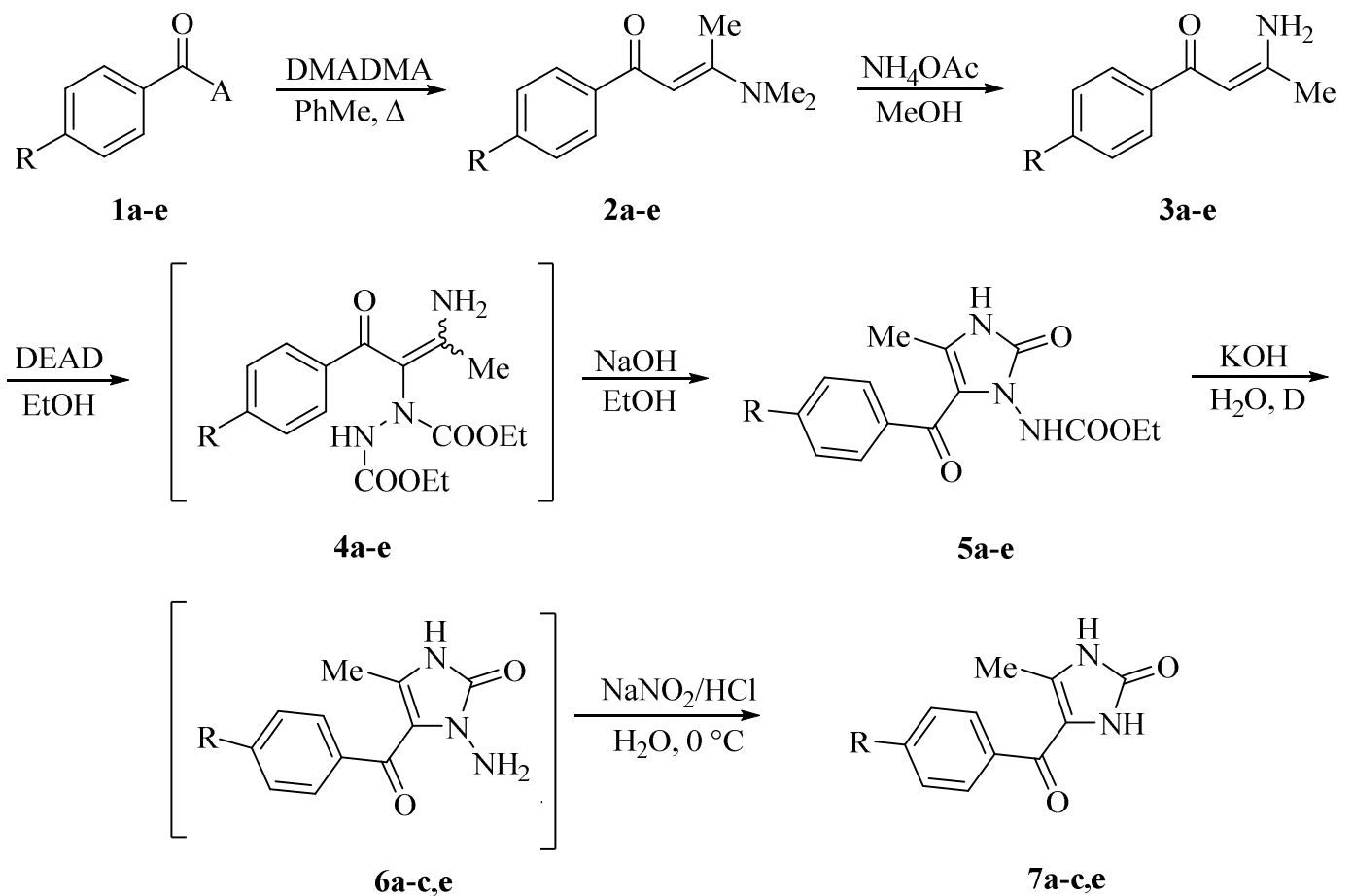

Scheme 1. 
Table 1. Yields of 4-aroyl-5-methyl-1H-imidazol-2(3H)-ones (7)

\begin{tabular}{c|c|c|c}
$\begin{array}{c}\text { Compounds } \\
\mathbf{1 - 7}\end{array}$ & $\mathbf{R}$ & Yield (\%) & Yield (\%) \\
\hline $\mathbf{a}$ & $\mathrm{SMe}$ & $\mathbf{1} \rightarrow \mathbf{7}(5)$ & $\mathbf{2} \rightarrow \mathbf{7}, 48$ \\
$\mathbf{b}$ & $\mathrm{H}$ & $\mathbf{1} \rightarrow \mathbf{7}(23)$ & \\
$\mathbf{c}$ & $\mathrm{Cl}$ & $\mathbf{1} \rightarrow \mathbf{7}(31)$ & \\
$\mathbf{d}$ & $\mathrm{SO}_{2} \mathrm{Me}$ & $/$ & $\mathbf{1} \rightarrow \mathbf{5}, 16$ \\
$\mathbf{e}$ & $\mathrm{Br}$ & $\mathbf{1} \rightarrow \mathbf{7}(27)$ & \\
$\mathbf{f}$ & $\mathrm{F}$ & $\mathbf{1} \rightarrow \mathbf{2}(67)$ & \\
\hline
\end{tabular}<smiles>CC(=O)c1ccc(F)cc1</smiles>


\section{Scheme 2}

The structure of (E)-1-aryl-3-(dimethylamino)but-2-en-1-ones 2a-e is supported by X-ray analysis for compound $\mathbf{2 d}$ (Figure 2). The orientation around the double bond in compounds 2 and $\mathbf{3}$ was determined on the basis of chemical shifts. In compounds $\mathbf{2}$ the methyl group appear downfield $(\delta=2.50-2.67 \mathrm{ppm})$ in comparison to the chemical shift of methyl group in compounds 3 ( $\delta=2.05-2.09 \mathrm{ppm})$. Furthermore, in (Z)-3-amino-1-aryl-but-2-en-1-ones (3) a large difference in chemical shifts $(\sim 5 \mathrm{ppm})$ for both protons attached to the amino group was observed, due to the strong intramolecular hydrogen bond of one proton to carbonyl group (Figure 2).

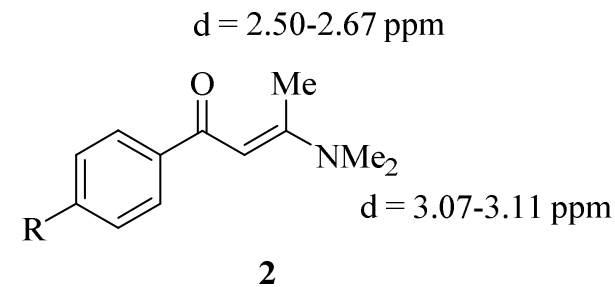

2

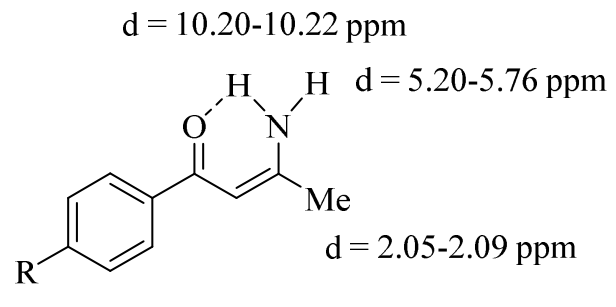

3

Figure 2. Characteristic ${ }^{1} \mathrm{H}$ chemical shifts for compounds $\mathbf{1}$ and $\mathbf{2}$. 


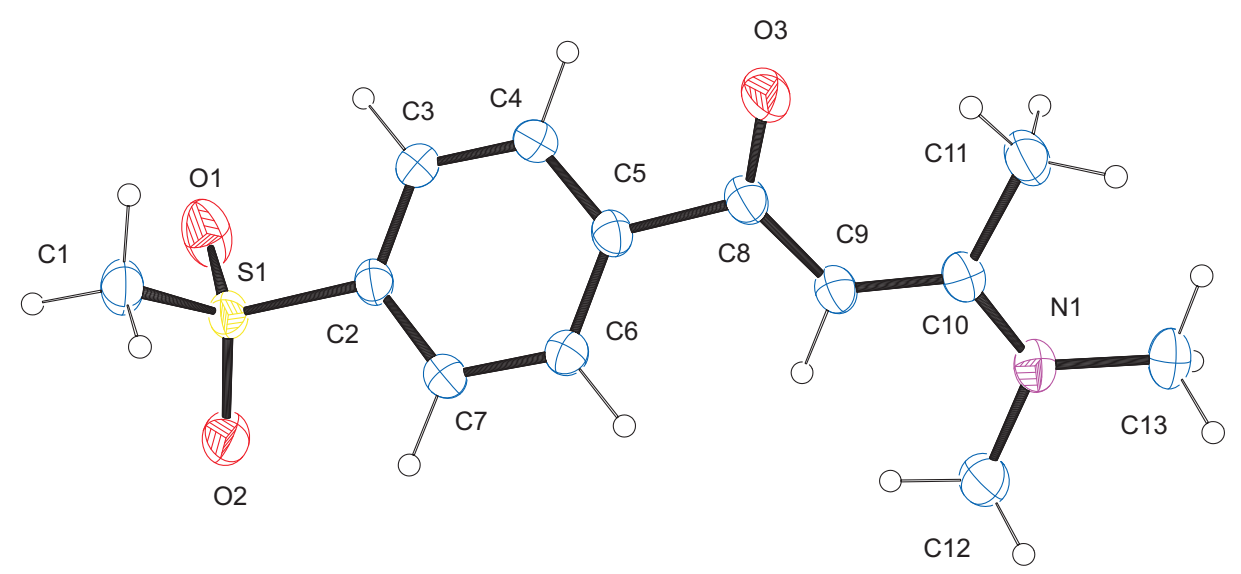

Figure 3. ORTEP plot of (E)-3-(dimethylamino)-1-((4-methylsulfonyl)phenyl)but-2-en-one (2d) at the $50 \%$ probability level of ellipsoids.

\section{Conclusions}

In summary, a new synthetic method for the preparation of 4-aroyl-5-methyl- $1 \mathrm{H}$-imidazol2(3H)-ones (7a-c,e) (Enoximone analogues) was developed. The synthesis started with commercially available methyl ketones 1a-f which were transformed with DMADMA into $(E)$ 1-aryl-3-(dimethylamino)-but-2-en-1-ones 2a-e. Substitution of the $N, N$-(dimethylamino) group with $\mathrm{NH}_{4} \mathrm{OAc}$ afforded the corresponding (Z)-3-amino-1-aryl-but-2-en-1-ones 3a-e. In the reaction of 3a-e with diethyl azodicarboxylate intermediates 4a-e were formed, which were cyclized into ethyl 5-aroyl-4-methyl-2-oxo-2,3-dihydro-1 $H$-imidazol-1-yl)carbamates 5a-e. Hydrolysis of the ester group, followed by the decarboxylation and deamination of 1-amino-5aroyl-4-methyl-1 $H$-imidazol-2(3H)-ones 6a-c,e as intermediates produced 4-aroyl-5-methyl- $1 H$ imidazol-2(3H)-ones 7a-c,e (Enoximone analogues).

\section{Experimental Section}

General. Melting points were determined on a Kofler micro hot stage and on a SRS OptiMelt MPA100-Automated Melting Point System. NMR spectra were obtained on a Bruker Avance DPX 300 at $300 \mathrm{MHz}$ for ${ }^{1} \mathrm{H}$ and $75.5 \mathrm{MHz}$ for ${ }^{13} \mathrm{C}$, and a Bruker UltraShield 500 plus at 500 $\mathrm{MHz}$ for ${ }^{1} \mathrm{H}$ and $126 \mathrm{MHz}$ for ${ }^{13} \mathrm{C}$, using DMSO- $d_{6}$ and $\mathrm{CDCl}_{3}$ with TMS as the internal standard, as solvents. Mass spectra were recorded on an AutoSpecQ spectrometer and Agilent 6224 Accurate Mass TOF LC/MS, IR spectra on a Perkin-Elmer Spectrum BX FTIR spectrophotometer. The microanalyses for $\mathrm{C}, \mathrm{H}$, and $\mathrm{N}$ were performed on a Perkin-Elmer $\mathrm{CHN}$ Analyser 2400 II. Column chromatography (CC) was performed on silica gel (Fluka, Silica gel 60, particle size: $0.035-0.070 \mathrm{~mm}$ ). 


\section{(E)-3-(Dimethylamino)-1-(4-(methylthio)phenyl)but-2-en-1-one (2a)}

Method A. 1-(4-(Methylthio)phenyl)ethanone (1a) (1.52 g, $9.1 \mathrm{mmol}$ ) was dissolved in dry PhMe $(200 \mathrm{~mL})$ and DMADMA $(1.3 \mathrm{~mL}, 9 \mathrm{mmol})$ was added. Reaction mixture was refluxed for $48 \mathrm{~h}$. Volatile components were evaporated and the product isolated by column chromatography (EtOAc/petroleum ether). Yield: $110 \mathrm{mg}(5.1 \%)$, yellow oil. ${ }^{1} \mathrm{H} \mathrm{NMR}\left(\mathrm{CDCl}_{3}\right.$, $500 \mathrm{MHz}): \delta 2.50\left(3 \mathrm{H}, \mathrm{s}, \mathrm{CH}_{3}\right) ; 2.66(3 \mathrm{H}, \mathrm{s}, \mathrm{SCH} 3) ; 3.07\left(6 \mathrm{H}, \mathrm{s}, \mathrm{NMe} e_{2}\right) ; 5.65(1 \mathrm{H}, \mathrm{s}, \mathrm{CH}) ; 7.22-$ $7.25(2 \mathrm{H}, \mathrm{m}, \mathrm{Ph}) ; 7.79-7.81(2 \mathrm{H}, \mathrm{m}, \mathrm{Ph}) .{ }^{13} \mathrm{C} \mathrm{NMR}\left(\mathrm{CDCl}_{3}, 126 \mathrm{MHz}\right): \delta 15.4,16.6,40.2,92.3$, $125.3,127.8,139.5,141.5,163.8,187.3$. EI-HRMS: $\mathrm{m} / z=236.1105\left(\mathrm{MH}^{+}\right) ; \mathrm{C}_{13} \mathrm{H}_{18} \mathrm{NOS}$ calculated: $\mathrm{m} / \mathrm{z}=236.1104\left(\mathrm{MH}^{+}\right) ; v_{\max }(\mathrm{KBr}) 3279,3143,1580,1548,1522,1485,1433,1400$, $1367,1323,1290,1271,1217,1184,1108,1092,1010,951,846 \mathrm{~cm}^{-1}$

Method B. (E)-3-(dimethylamino)-1-(4-fluorophenyl)but-2-en-1-one (2f) (2 mmol, $412 \mathrm{mg}$ ) was dissolved in dry DMF (3 mL) and MeSNa (2 mmol, $140 \mathrm{mg}$ ) was added. Reaction mixture was stirred for $24 \mathrm{~h}$ at $70{ }^{\circ} \mathrm{C}$. Volatile components were evaporated and the product isolated by column chromatography (EtOAC/petroleum ether =1:1). Yield: $376 \mathrm{mg}(80 \%)$. ${ }^{1} \mathrm{H}$ NMR is in agreement with ${ }^{1} \mathrm{H}$ NMR spectra of the product prepared by the method A.

\section{(E)-3-(Dimethylamino)-1-(4-(methylsulfonyl)phenyl)but-2-en-1-one (2d)}

1-(4-(Methylsulfonyl)phenyl)ethanone (1d) (1.98 g, $10 \mathrm{mmol})$ was dissolved in dry PhMe (20 $\mathrm{mL})$ and DMADMA $(2.19 \mathrm{~mL}, 15 \mathrm{mmol})$ was added to reaction mixture. Reaction mixture was refluxed for $3 \mathrm{~h}$. Upon cooling the product precipitated from reaction mixture and was filtered of. Recrystallization from EtOAc. Yield: $1.69 \mathrm{~g}(63 \%)$, yellow solid, mp 168.1-170.0 ${ }^{\circ} \mathrm{C} .{ }^{1} \mathrm{H}$ NMR $\left(\mathrm{CDCl}_{3}, 300 \mathrm{MHz}\right): \delta 2.67\left(3 \mathrm{H}, \mathrm{s}, \mathrm{CH}_{3}\right) ; 3.12(3 \mathrm{H}, \mathrm{s}, \mathrm{CH}) ; 3.11\left(6 \mathrm{H}, \mathrm{s}, \mathrm{NMe} e_{2}\right) ; 5.60(1 \mathrm{H}, \mathrm{s}$, $\mathrm{CH}) ; 7.92-8.00(4 \mathrm{H}, \mathrm{m}, \mathrm{Ph}) .{ }^{13} \mathrm{C} \mathrm{NMR}\left(\mathrm{CDCl}_{3}, 75.5 \mathrm{MHz}\right): \delta 16.7,40.4,44.5,92.3,127.2$, 128.1, 141.3, 148.2, 165.4, 185.8. $\left(\mathrm{C}_{13} \mathrm{H}_{17} \mathrm{NO}_{3} \mathrm{~S}\right.$ calculated: $\mathrm{C}, 58.40 ; \mathrm{H}, 6.41 ; \mathrm{N}, 5.24$. found $\mathrm{C}$, 58.41; H, 6.58; N, 5.20); EI-HRMS: $m / z=268.1003\left(\mathrm{MH}^{+}\right) ; \mathrm{C}_{13} \mathrm{H}_{18} \mathrm{NO}_{3} \mathrm{~S}$ calculated: $\mathrm{m} / \mathrm{z}=$ $268.1007\left(\mathrm{MH}^{+}\right)$; $v_{\max }(\mathrm{KBr}) 3011,2997,2917,1608,1538,1477,1434,1385,1353,1306,1290$, $1223,1179,1153,1082,1026,973,918,863,851 \mathrm{~cm}^{-1}$.

\section{(E)-1-(4-Bromophenyl)-3-(dimethylamino)but-2-en-1-one (2e) ${ }^{29}$}

1-(4-Bromophenyl)ethanone (1e) $(4.00 \mathrm{~g}, 20 \mathrm{mmol})$ was dissolved in dry $\mathrm{PhMe}(50 \mathrm{~mL})$ and DMADMA (3.8 mL, $26 \mathrm{mmol}$ ) was added. The reaction mixture was refluxed for $3 \mathrm{~h}$ and chilled. When petroleum ether was added the product precipitated from the reaction mixture and was recrystallized from EtOAc. Yield: $2.81 \mathrm{~g}(53 \%)$, yellow solid, mp 118.8-120.4 ${ }^{\circ} \mathrm{C} .{ }^{1} \mathrm{H}$ NMR $\left(\mathrm{CDCl}_{3}, 300 \mathrm{MHz}\right): \delta 2.65\left(3 \mathrm{H}, \mathrm{s}, \mathrm{CH}_{3}\right) ; 3.08(6 \mathrm{H}, \mathrm{s}, \mathrm{NMe}) ; 5.60(1 \mathrm{H}, \mathrm{s}, \mathrm{CH}) ; 7.48-7.53(2 \mathrm{H}, \mathrm{m}$, $\mathrm{Ph}) ; 7.70-7.74(2 \mathrm{H}, \mathrm{m}, \mathrm{Ph}) .{ }^{13} \mathrm{C} \mathrm{NMR}\left(\mathrm{CDCl}_{3}, 75.5 \mathrm{MHz}\right): \delta 16.8,40.4,92.4,124.9,129.1$, 131.4, 142.1, 164.6, 187.0.

\section{(E)-3-(Dimethylamino)-1-(4-fluorophenyl)but-2-en-1-one (2f) ${ }^{29}$}

1-(4-Fluorophenyl)ethanone (1f) $(6.06 \mathrm{~mL}, 50 \mathrm{mmol})$ was dissolved in dry PhMe $(100 \mathrm{~mL})$ and DMADMA (11.00 mL, $75 \mathrm{mmol}$ ) was added to reaction mixture. Reractiom mixture was 
refluxed for $48 \mathrm{~h}$ volatile components were evaporated and the product was isolated by column chromatography (EtOAC/petroleum ether = 1:1). Yield: $6.93 \mathrm{~g}(67 \%)$, yellow solid. ${ }^{1} \mathrm{H}$ NMR $\left.\left(\mathrm{CDCl}_{3}, 500 \mathrm{MHz}\right): \delta 2.65\left(3 \mathrm{H}, \mathrm{s}, \mathrm{CH}_{3}\right) ; 3.08(6 \mathrm{H}, \mathrm{s}, \mathrm{NMe})_{2}\right) ; .62(1 \mathrm{H}, \mathrm{s}, \mathrm{CH}) ; 7.03-7.07(2 \mathrm{H}, \mathrm{m}$, $\mathrm{Ph}) ; 7.84-7.88(2 \mathrm{H}, \mathrm{m}, \mathrm{Ph})$.

\section{(Z)-3-Amino-1-(4-(methylthio)phenyl)but-2-en-1-one (3a)}

(E)-3-(Dimethylamino)-1-(4-(methylthio)phenyl)but-2-en-1-one (2a) (108 $\mathrm{mg}, 0.46 \mathrm{mmol}$ ) was dissolved in $\mathrm{MeOH}(3 \mathrm{~mL}) \mathrm{NH}_{4} \mathrm{OAc}(308 \mathrm{mg}, 4 \mathrm{mmol})$ was added and the reaction mixture stirred for $18 \mathrm{~h}$ at room temperature. Volatile components were evaporated and the product isolated by column chromatography $(\mathrm{EtOAc} /$ petroleum ether $=1: 1)$. Recrystallization from MeOH. Yield: $95 \mathrm{mg}$ (99\%), yellow solid, mp 154.0-156.2 ${ }^{\circ} \mathrm{C} .{ }^{1} \mathrm{H}$ NMR $\left(\mathrm{CDCl}_{3}, 500 \mathrm{MHz}\right): \delta$ $2.05\left(3 \mathrm{H}, \mathrm{s}, \mathrm{CH}_{3}\right) ; 2.51\left(3 \mathrm{H}, \mathrm{s}, \mathrm{SCH}_{3}\right) ; 5.20\left(1 \mathrm{H}\right.$, br s, $\mathrm{NH}$ from $\left.\mathrm{NH}_{2}\right) ; 5.71(1 \mathrm{H}, \mathrm{s}, \mathrm{CH}) ; 7.24-$ $7.26(2 \mathrm{H}, \mathrm{m}, \mathrm{Ph}) ; 7.80-7.82(2 \mathrm{H}, \mathrm{m}, \mathrm{Ph}) ; 10.20\left(1 \mathrm{H}\right.$, br s, $\mathrm{NH}$ from $\left.\mathrm{NH}_{2}\right) .{ }^{13} \mathrm{C} \mathrm{NMR}\left(\mathrm{CDCl}_{3}, 126\right.$ $\mathrm{MHz}): \delta 15.3,23.1,92.1,125.4,127.7,136.7,142.5,162.8,188.7 .\left(\mathrm{C}_{11} \mathrm{H}_{13} \mathrm{NOS}\right.$ calculated: $\mathrm{C}$, 63.74; H, 6.23; N, 6.76. found C, 63.53; H, 6.27; N, 6.70); EI-HRMS: $m / z=208.0790\left(\mathrm{MH}^{+}\right)$; $\mathrm{C}_{11} \mathrm{H}_{14} \mathrm{NOS}$ calculated: $\mathrm{m} / \mathrm{z}=208.00791\left(\mathrm{MH}^{+}\right) ; v_{\max }(\mathrm{KBr}) 3280,3140,1600,1583,1559,157$, $1477,1443,1396,1370,1314,1288,273,1171,1119,1104,1069,1007,845 \mathrm{~cm}^{-1}$.

\section{(Z)-3-Amino-1-(4-(methylsulfonylphenyl)but-2-en-1-one (3d)}

(E)-3-(dimethylamino)-1-(4-(methylsulfonylphenyl)but-2-en-1-one (2d) (1.5 g, $5.65 \mathrm{mmol})$ was dissolved in $\mathrm{MeOH}(30 \mathrm{~mL}) \mathrm{NH}_{4} \mathrm{OAc}$ was added $(4.35 \mathrm{~g}, 50 \mathrm{mmol})$ and reaction mixture stirred for $14 \mathrm{~h}$ at room temperature. Volatile components were evaporated and the product was isolated by column chromatography (EtOAc). Recrystallization from EtOAc/petroleum ether. Yield: 1.33 g (99 \%), yellow solid, mp 150.2-151.3 ${ }^{\circ} \mathrm{C} .{ }^{1} \mathrm{H}$ NMR $\left(\mathrm{CDCl}_{3}, 300 \mathrm{MHz}\right): \delta 2.09\left(3 \mathrm{H}, \mathrm{s}, \mathrm{CH}_{3}\right)$; $3.08\left(3 \mathrm{H}, \mathrm{s}, \mathrm{CH}_{3}\right) ; 5.71(1 \mathrm{H}, \mathrm{s}, \mathrm{CH}) ; 5.76\left(1 \mathrm{H}\right.$, br s, $\mathrm{NH}$ from $\left.\mathrm{NH}_{2}\right) ; 7.95-8.04(4 \mathrm{H}, \mathrm{m}, \mathrm{Ph}) ; 10.32$ $\left(1 \mathrm{H}\right.$, br s, $\mathrm{NH}$ from $\left.\mathrm{NH}_{2}\right) .{ }^{13} \mathrm{C} \mathrm{NMR}\left(\mathrm{CDCl}_{3}, 75.5 \mathrm{MHz}\right): \delta 23.2,44.8,92.8,127.7,128.3,142.2$, 145.5, 165.3, 187.2. $\left(\mathrm{C}_{11} \mathrm{H}_{13} \mathrm{NO}_{3} \mathrm{~S}\right.$ calculated: $\mathrm{C}, 55.21 ; \mathrm{H}, 5.48 ; \mathrm{N}, 5.85$. found $\mathrm{C}, 55.18 ; \mathrm{H}$, 5.33; N, 5.79); EI-HRMS: $m / z=240.0699\left(\mathrm{MH}^{+}\right) ; \mathrm{C}_{11} \mathrm{H}_{14} \mathrm{NO}_{3} \mathrm{~S}$ calculated: $\mathrm{m} / \mathrm{z}=240.0694$ $\left(\mathrm{MH}^{+}\right) ; v_{\max }(\mathrm{KBr}) 3282,3142,1617,1560,1531,1398,1319,1307,1288,1214,1178,1151$, $1106,1014,962,846 \mathrm{~cm}^{-1}$.

\section{(Z)-3-Amino-1-(4-bromophenyl)but-2-en-1-one (3e)}

(Z)-1-(4-bromophenyl)-3-(dimethylamino)but-2-en-1-one (2e) (2.81 g, $10.5 \mathrm{mmol})$ was dissolved in $\mathrm{MeOH}(50 \mathrm{~mL}) \mathrm{NH}_{4} \mathrm{OAc}$ was added $(7.7 \mathrm{~g}, 100 \mathrm{mmol})$ and the reaction mixture was stirred for $2 \mathrm{~h}$ at room temperature. Volatile components were evaporated and the product was isolated by column chromatography $($ EtOAc/petroleum ether $=1: 1)$. Recrystallization from EtOAc/petroleum ether. Yield: $2.47 \mathrm{~g}$ (99\%), yellow solid, $\mathrm{mp} 129.6-131.2{ }^{\circ} \mathrm{C}\left(\mathrm{mp}^{30}=126-128\right.$ $\left.{ }^{\circ} \mathrm{C}\right) .{ }^{1} \mathrm{H} \mathrm{NMR}\left(\mathrm{CDCl}_{3}, 300 \mathrm{MHz}\right): \delta 2.06\left(3 \mathrm{H}, \mathrm{s}, \mathrm{CH}_{3}\right) ; 5.27\left(1 \mathrm{H}\right.$, br s, $\mathrm{NH}$ from $\left.\mathrm{NH}_{2}\right) ; 5.67(1 \mathrm{H}$, $\mathrm{s}, \mathrm{CH}) ; 7.51-7.55(2 \mathrm{H}, \mathrm{m}, \mathrm{Ph}) ; 7.72-7.76(2 \mathrm{H}, \mathrm{m}, \mathrm{Ph}) ; 10.22\left(1 \mathrm{H}\right.$, br s, $\mathrm{NH}$ from $\left.\mathrm{NH}_{2}\right) .{ }^{13} \mathrm{C} \mathrm{NMR}$ $\left(\mathrm{CDCl}_{3}, 75.5 \mathrm{MHz}\right): \delta 23.1,92.2,125.7,129.0,131.6,139.2,163.6,188.3 .\left(\mathrm{C}_{10} \mathrm{H}_{10} \mathrm{NOBr}\right.$ 
calculated: C, 50.02; H, 4.20; N, 5.83. found $\mathrm{C}, 50.00 ; \mathrm{H}, 4.10 ; \mathrm{N}, 5.82) ;$ EI-HRMS: $m / z=$ $240.0019\left(\mathrm{MH}^{+}\right) ; \mathrm{C}_{11} \mathrm{H}_{11} \mathrm{NOBr}$ calculated: $\mathrm{m} / \mathrm{z}=240.0019\left(\mathrm{MH}^{+}\right) ; v_{\max }(\mathrm{KBr}) 3280,3140,1600$, 1583, 1559, 157, 1477, 1443, 1396, 1370, 1314, 1288, 273, 1171, 1119, 1104, 1069, 1007, 845 $\mathrm{cm}^{-1}$.

\section{Diethyl 1-(3-amino-1-(4-(methylsulfonylphenyl)-1-oxobut-2-en-2-yl)hydrazine-1,2- dicarboxylate (4d)}

(Z)-3-amino-1-(4-(methylsulfonylphenyl)but-2-en-1-one (3d) $\quad(570 \quad \mathrm{mg}, \quad 2.38 \mathrm{mmol})$ was dissolved in MeCN (10 mL) DEAD was added (408 $\mu \mathrm{L}, 2.6 \mathrm{mmol})$ and reaction mixture stirred for $12 \mathrm{~h}$ at room temperature Volatile components were evaporated and the product was isolated by column chromatography (EtOAc/petroleum ether $=1: 1)$. Yield: $450 \mathrm{mg}(46 \%)$, yellow oil. ${ }^{1} \mathrm{H}$ NMR $\left(\mathrm{CDCl}_{3}, 500 \mathrm{MHz}\right): \delta 1.17-1.27(6 \mathrm{H}, \mathrm{m} \mathrm{2xCH}) ; 2.39\left(3 \mathrm{H}, \mathrm{s}, \mathrm{CH}_{3}\right) ; 3.06\left(3 \mathrm{H}, \mathrm{s}, \mathrm{CH}_{3}\right)$; 4.02-4.27 (4H, m, 2xCH $\mathrm{CH}_{2}$; $5.66(1 \mathrm{H}$, br s, $\mathrm{NH})$; 5.72-5.88 (1H, m, NH); 7.49-7.54 (1H, m, Ph); 7.57-7.62 (1H, m, Ph); 7.94-8.00 $(2 \mathrm{H}, \mathrm{m}, \mathrm{Ph})$; 10.42-10.59 $(1 \mathrm{H}, \mathrm{m}, \mathrm{NH})$. EI-HRMS: $m / z=$ $414.1329\left(\mathrm{MH}^{+}\right) ; \mathrm{C}_{17} \mathrm{H}_{24} \mathrm{~N}_{3} \mathrm{O}_{7} \mathrm{~S}$ calculated: $\mathrm{m} / \mathrm{z}=414.1329\left(\mathrm{MH}^{+}\right) ; v_{\max }(\mathrm{NaCl}) 3382,2982$, 2919, 2357, 1716, 1610, 1478, 1393, 1375, 1341, 1284, 1236, 1155, 1135, 1069, 1088, 958, 908, $841 \mathrm{~cm}^{-1}$.

Diethyl 1-(3-amino-1-(4-bromophenyl)-1-oxobut-2-en-2-yl)hydrazine-1,2-dicarboxylate (4e) (Z)-3-amino-1-(4-bromophenyl)but-2-en-1-one (3e) $(670 \mathrm{mg}, 2.80 \mathrm{mmol})$ was dissolved in $\mathrm{MeCN}(10 \mathrm{~mL}) \mathrm{DEAD}$ was added $(486 \mu \mathrm{L}, 3.1 \mathrm{mmol})$ and the reaction mixture was stirred $14 \mathrm{~h}$ at room temperature. Volatile components were evaporated and the product was isolated by column chromatography (EtOAc/petroleum ether $=1: 1)$. Yield: $1.00 \mathrm{mg}(87 \%)$, yellow oil. ${ }^{1} \mathrm{H}$ NMR $\left(\mathrm{CDCl}_{3}, 500 \mathrm{MHz}\right): \delta 1.18-1.26(6 \mathrm{H}, \mathrm{m}, 2 \mathrm{xCH}) ; 2.33-2.40\left(3 \mathrm{H}, \mathrm{s}, \mathrm{CH}_{3}\right) ; 4.05-4.30(4 \mathrm{H}$, $\left.\mathrm{m}, 2 \mathrm{xCH})_{2}\right) 5.53(1 \mathrm{H}$, br s, $\mathrm{NH}) ; 5.81-5.86(1 \mathrm{H}, \mathrm{m}, \mathrm{NH}) ; 7.17-7.21(1 \mathrm{H}, \mathrm{m}, \mathrm{Ph}) ; 7.26-7.63(1 \mathrm{H}$,

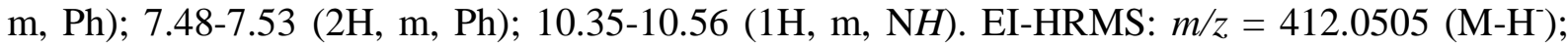
$\mathrm{C}_{16} \mathrm{H}_{19} \mathrm{~N}_{3} \mathrm{O}_{5} \mathrm{Br}$ calculated: $\mathrm{m} / \mathrm{z}=412.0514\left({\left.\mathrm{M}-\mathrm{H}^{-}\right)}^{-} v_{\max }(\mathrm{NaCl}) 3354,2981,2925,2358,1713\right.$, $1608,1584,1480,1374,1320,1286,1228,1140,1093,1070,1012,861,837 \mathrm{~cm}^{-1}$.

\section{Ethyl [4-methyl-5-(4-(methylthiobenzoyl)-2-oxo-2,3-dihydro-1H-imidazol-1-yl]carbamate} (5a)

(Z)-3-amino-1-(4-(methylthiophenyl)but-2-en-1-one (3a) $(93 \mathrm{~g}, 0.45 \mathrm{mmol})$ was dissolved in (2 $\mathrm{mL})$ DEAD $(80 \mu \mathrm{L}, 0.5 \mathrm{mmol})$ was added and reaction mixture stirred for $2 \mathrm{~h}$ at room temperature. Intermediate diethyl 1-(3-amino-1-(4-(methylthio)phenyl)-1-oxobut-2-en-2yl)hydrazine-1,2-dicarboxylate (4a) was used directly in the cyclisation step without purification. $\mathrm{NaOH}(60 \mathrm{mg}, 1.5 \mathrm{mmol}$ ) was added to the reaction mixture and it was stirred for $48 \mathrm{~h}$ at room temperature. Volatile components were evaporated and the product isolated by column chromatography $\left(\mathrm{CHCl}_{3} / \mathrm{MeOH}=15: 1\right)$. Yield: $103 \mathrm{mg}(68 \%)$, white solid, mp 234.3-237.4 ${ }^{\circ} \mathrm{C}$. ${ }^{1} \mathrm{H}$ NMR (DMSO- $\left.d_{6}, 500 \mathrm{MHz}\right): \delta 1.15\left(3 \mathrm{H}, \mathrm{t}, J=7.1 \mathrm{~Hz}, \mathrm{CH}_{3}\right) ; 1.85\left(3 \mathrm{H}, \mathrm{s}, \mathrm{CH}_{3}\right) ; 2.53(3 \mathrm{H}, \mathrm{s}$, $\left.\mathrm{SCH}_{3}\right) ; 4.02\left(2 \mathrm{H}, \mathrm{s}, \mathrm{J}=7.1 \mathrm{~Hz}, \mathrm{CH}_{2}\right) ; 7.33-7.35(2 \mathrm{H}, \mathrm{m}, \mathrm{Ph}) ; 7.56-7.58(2 \mathrm{H}, \mathrm{m}, \mathrm{Ph}) ; 9.85(1 \mathrm{H}, \mathrm{s}$, 
$\mathrm{N} H) ; 11.12\left(1 \mathrm{H}\right.$, br s, NH). ${ }^{13} \mathrm{C}$ NMR (DMSO-d $\left.6,126 \mathrm{MHz}\right): \delta 12.2,14.0,14.4,61.0,119.7$, 124.8, 128.8, 129.3, 134.9, 144.4, 151.8, 155.4, 182.5. EI-HRMS: $m / z=336.1008\left(\mathrm{MH}^{+}\right)$; $\mathrm{C}_{15} \mathrm{H}_{18} \mathrm{~N}_{3} \mathrm{O}_{4} \mathrm{~S}$ calculated: $\mathrm{m} / \mathrm{z}=336.1013\left(\mathrm{MH}^{+}\right) ; v_{\max }(\mathrm{KBr}) 3185,2991,1740,1723,1686$, $1625,1585,1542$, 1431, 1368, 1350, 1272, 1243, 1201, 1180, 1111, 1086, 1064, 1019, 978, 957, $914,864,829 \mathrm{~cm}^{-1}$.

Ethyl [5-(4-chlorobenzoyl)-4-methyl-2-oxo-2,3-dihydro-1H-imidazol-1-yl]carbamate (5c) (Z)-3-amino-1-(4-chlorophenyl)but-2-en-1-one ${ }^{30}$ (3c) (3.74 g, $19.2 \mathrm{mmol}$ ) was dissolved in EtOH $(50 \mathrm{~mL})$ DEAD $(3.57 \mathrm{~mL}, 22 \mathrm{mmol})$ was added and the reaction mixture was stirred for 4 $\mathrm{h}$ at room temperature. Intermediate diethyl 1-(3-amino-1-(4-chlorophenyl)-1-oxobut-2-en-2yl)hydrazine-1,2-dicarboxylate (4c) was directly used in next step without isolation. To $\mathbf{4 c}$ was added $\mathrm{NaOH}(1.6 \mathrm{~g}, 40 \mathrm{mmol})$ and reaction mixture was stirred for $15 \mathrm{~h}$ at room temperature. Product 5c was isolated by column chromatography $\left(\mathrm{CHCl}_{3} / \mathrm{MeOH}=8: 1\right)$. Yield: $4.73 \mathrm{~g}(76 \%)$, white solid, mp 238.3-240.0 ${ }^{\circ} \mathrm{C} .{ }^{1} \mathrm{H}$ NMR (DMSO- $\left.d_{6}, 500 \mathrm{MHz}\right): \delta 1.15\left(3 \mathrm{H}, \mathrm{t}, J=7.1 \mathrm{~Hz}, \mathrm{CH}_{3}\right.$ ); $1.85\left(3 \mathrm{H}, \mathrm{s}, \mathrm{CH}_{3}\right) ; 4.02\left(2 \mathrm{H}, \mathrm{s}, \mathrm{J}=7.1 \mathrm{~Hz}, \mathrm{CH}_{2}\right)$; 7.55-7.59 (2H, m, Ph); 7.61-7.66 (2H, m, Ph);

$9.87(1 \mathrm{H}, \mathrm{s}, \mathrm{N} H) ; 11.25\left(1 \mathrm{H}\right.$, br s, NH). ${ }^{13} \mathrm{C} \mathrm{NMR}$ (DMSO- $\left.d_{6}, 126 \mathrm{MHz}\right): \delta 12.2,14.4,61.0$, $119.5,128.7,130.3,130.4,137.0,137.7,151.7,155.4,182.2 .\left(\mathrm{C}_{14} \mathrm{H}_{14} \mathrm{~N}_{3} \mathrm{O}_{4} \mathrm{Cl}\right.$ calculated: $\mathrm{C}$, 51.94; H, 4.36; N, 12.98. found C, 51.93; H, 4.14; N, 12.98); EI-HRMS: $m / z=324.0748\left(\mathrm{MH}^{+}\right)$; $\mathrm{C}_{14} \mathrm{H}_{15} \mathrm{~N}_{3} \mathrm{O}_{4} \mathrm{Cl}$ calculated: $\mathrm{m} / \mathrm{z}=324.0746\left(\mathrm{MH}^{+}\right) ; v_{\max }(\mathrm{KBr}) 3193,3067,3005,2982,1738$, 1722 , 1687, 1619, 1585, 1536, 1474, 1435, 1412, 1369, 1344, 1279, 1261, 1197, 1177, 1109, $1065,1011,980,956,914,870,833 \mathrm{~cm}^{-1}$.

\section{Ethyl}

[4-methyl-5-(4-(methylsulfonyl)benzoyl)-2-oxo-2,3-dihydro-1H-imidazol-1yl]carbamate (5d)

Diethyl 1-(3-amino-1-(4-(methylsulfonyl)phenyl)-1-oxobut-2-en-2-yl)hydrazine-1,2-dicarboxylate (4d) $(450 \mathrm{mg}, 1.09 \mathrm{mmol})$ was dissolved in $\mathrm{EtOH}(5 \mathrm{~mL})$ and $\mathrm{NaOH}$ was added $(110 \mathrm{mg}$, $2.18 \mathrm{mmol})$. Reaction mixture was stirred for $15 \mathrm{~h}$ at room temperature and product isolated by column chromatography $\left(\mathrm{CHCl}_{3} / \mathrm{MeOH}=10: 1\right)$. Yield: $220 \mathrm{mg}(55 \%)$, yellow solid, mp 196.5$201.1{ }^{\circ} \mathrm{C} .{ }^{1} \mathrm{H}$ NMR (DMSO-d $\left.6,500 \mathrm{MHz}\right): \delta 1.14\left(3 \mathrm{H}, \mathrm{t}, J=7.0 \mathrm{~Hz}, \mathrm{CH}_{3}\right) ; 1.85\left(3 \mathrm{H}, \mathrm{s}, \mathrm{CH}_{3}\right)$; $3.28\left(3 \mathrm{H}, \mathrm{s}, \mathrm{CH}_{3}\right) ; 3.97-4.04\left(2 \mathrm{H}, \mathrm{m}, \mathrm{CH}_{2}\right) ; 7.81(2 \mathrm{H}, \mathrm{d}, J=8.0 \mathrm{~Hz}, \mathrm{Ph}) ; 8.03(2 \mathrm{H}, \mathrm{d}, J=8.0 \mathrm{~Hz}$, $\mathrm{Ph}) ; 9.88(1 \mathrm{H}, \mathrm{s}, \mathrm{N} H) ; 11.36(1 \mathrm{H}, \mathrm{br} \mathrm{s}, \mathrm{N} H) .{ }^{13} \mathrm{C}$ NMR (DMSO- $\left.d_{6}, 126 \mathrm{MHz}\right): \delta 12.4,14.4,43.3$, 61.1, 119.3, 127.3, 129.1, 131.7, 143.2, 151.6, 155.4, 182.1. $\left(\mathrm{C}_{15} \mathrm{H}_{17} \mathrm{~N}_{3} \mathrm{O}_{6}\right.$ calculated: $\mathrm{C}, 49.04$; $\mathrm{H}, 4.66 ; \mathrm{N}, 11.44$. found $\mathrm{C}, 49.08 ; \mathrm{H}, 4.59 ; \mathrm{N}, 11.20)$; EI-HRMS: $\mathrm{m} / z=368.0900\left(\mathrm{MH}^{+}\right)$; $\mathrm{C}_{15} \mathrm{H}_{18} \mathrm{~N}_{3} \mathrm{O}_{6} \mathrm{~S}$ calculated: $\mathrm{m} / \mathrm{z}=368.0911\left(\mathrm{MH}^{+}\right) ; v_{\max }(\mathrm{KBr}) 3412,1742,1713,1626,1430$, $1315,1266,1198,1153,1117,1089,1064,1013,967,907,870,768,756,694 \mathrm{~cm}^{-1}$.

\section{Ethyl [5-(4-bromobenzoyl)-4-methyl-2-oxo-2,3-dihydro-1H-imidazol-1-yl]carbamate (5e)}

Diethyl 1-(3-amino-1-(4-bromophenyl)-1-oxobut-2-en-2-yl)hydrazine-1,2-dicarboxylate (4e) $(1.00 \mathrm{~g}, 2.44 \mathrm{mmol})$ was dissolved in $\mathrm{EtOH}(10 \mathrm{~mL})$ and $\mathrm{NaOH}$ was added $(260 \mathrm{mg}, 6.5 \mathrm{mmol})$. Reaction mixture was stirred for $14 \mathrm{~h}$ at room temperature and product isolated by column 
chromatography $\left(\mathrm{CHCl}_{3} / \mathrm{MeOH}=10: 1\right)$. Yield: $610 \mathrm{mg}(68 \%)$, white solid, $\mathrm{mp} 247.7-249.9{ }^{\circ} \mathrm{C}$. ${ }^{1} \mathrm{H}$ NMR (DMSO- $\left.d_{6}, 500 \mathrm{MHz}\right): \delta 1.14\left(3 \mathrm{H}, \mathrm{t}, J=7.1 \mathrm{~Hz}, \mathrm{CH}_{3}\right) ; 1.84\left(3 \mathrm{H}, \mathrm{s}, \mathrm{CH}_{3}\right) ; 4.01(2 \mathrm{H}, \mathrm{q}$, $\left.J=7.1 \mathrm{~Hz}, \mathrm{CH}_{2}\right) ; 7.54(2 \mathrm{H}, \mathrm{d}, J=8.4 \mathrm{~Hz}, \mathrm{Ph}) ; 7.70(2 \mathrm{H}, \mathrm{d}, J=8.4 \mathrm{~Hz}, \mathrm{Ph}) ; 9.85(1 \mathrm{H}, \mathrm{s}, \mathrm{NH})$; $11.24(1 \mathrm{H}$, br s, $\mathrm{NH}) .{ }^{13} \mathrm{C}$ NMR (DMSO- $d_{6}, 126 \mathrm{MHz}$ ): $\delta 12.3,14.4,61.0,119.4,126.0,130.4$, 130.5, 131.7, 138.0, 151.6, 155.4, 182.3. $\left(\mathrm{C}_{14} \mathrm{H}_{14} \mathrm{~N}_{3} \mathrm{O}_{4} \mathrm{Br}\right.$ calculated: $\mathrm{C}, 45.67 ; \mathrm{H}, 3.83 ; \mathrm{N}, 11.41$. found $\mathrm{C}$, 45.91; $\mathrm{H}, 3.61 ; \mathrm{N}, 11.26)$; EI-HRMS: $m / z=368.0236\left(\mathrm{MH}^{+}\right) ; \mathrm{C}_{14} \mathrm{H}_{15} \mathrm{~N}_{3} \mathrm{O}_{4} \mathrm{Br}$ calculated: $\mathrm{m} / \mathrm{z}=368.0240\left(\mathrm{MH}^{+}\right) ; v_{\max }(\mathrm{KBr}) 3414,3240,1744,1692,1637,1584,1540,1418$, $1283,1196,1179,1068,1010,981,914,869 \mathrm{~cm}^{-1}$.

\section{5-Methyl-4-(4-(methylthio)benzoyl)-1H-imidazol-2(3H)-one or Enoxymone (7a)}

Ethyl (4-methyl-5-(4-(methylthio)benzoyl)-2-oxo-2,3-dihydro-1H-imidazol-1-yl)carbamate (5a) (95 mg, $0.28 \mathrm{mmol}$ ) was dissolved in $\mathrm{EtOH}(5 \mathrm{~mL}) \mathrm{KOH}$ was added (220 mg, $3.4 \mathrm{mmol})$ and the reaction mixture was reflxed for $20 \mathrm{~h}$. Volatile components were evaporated the solid residue dissolved in $\mathrm{H}_{2} \mathrm{O}(1 \mathrm{~mL})$ and concentrated solution of $\mathrm{HCl}_{(\mathrm{aq})}(3 \mathrm{~mL})$ was added. Reaction mixture was than chilled to $0{ }^{\circ} \mathrm{C}$ and $2 \mathrm{M} \mathrm{NaNO}_{2(\mathrm{aq})}(5 \mathrm{~mL})$ was slowly added during $20 \mathrm{~min}$. When all $\mathrm{NaNO}_{2(\mathrm{aq})}$ was added the reaction mixture was stirred further for $20 \mathrm{~min}$ at room temperature. White solid precipitated which was filtered and washed with $\mathrm{Et}_{2} \mathrm{O}(3 \times 5 \mathrm{~mL})$. Yield: $65 \mathrm{mg}$ (93\%), white solid, mp 251.0-254.3 ${ }^{\circ} \mathrm{C}\left(\mathrm{mp}^{17} 255-258{ }^{\circ} \mathrm{C}\right) .{ }^{1} \mathrm{H}$ NMR (DMSO- $d_{6}, 500$ $\mathrm{MHz}): \delta 1.89\left(3 \mathrm{H}, \mathrm{s}, \mathrm{CH}_{3}\right) ; 2.53\left(3 \mathrm{H}, \mathrm{s}, \mathrm{SCH}_{3}\right) ; 7.32-7.34(2 \mathrm{H}, \mathrm{m}, \mathrm{Ph}) ; 7.55-7.57$ (2H, m, Ph); $10.27\left(1 \mathrm{H}\right.$, br s, NH); $10.85\left(1 \mathrm{H}\right.$, br s, NH). ${ }^{13} \mathrm{C}$ NMR (DMSO- $\left.d_{6}, 126 \mathrm{MHz}\right): \delta 12.2,14.0$, 119.0, 124.8, 128.9, 131.5, 135.1, 143.3, 152.8, 182.8. EI-HRMS: $m / z=249.0690\left(\mathrm{MH}^{+}\right)$; $\mathrm{C}_{12} \mathrm{H}_{13} \mathrm{~N}_{2} \mathrm{O}_{2} \mathrm{~S}$ calculated: $\mathrm{m} / \mathrm{z}=249.0692\left(\mathrm{MH}^{+}\right) ; v_{\max }(\mathrm{KBr}) 3156,6023,2917,2854,1744$, 1694, 1610, 1588, 1547, 1488, 1465, 1434, 1372, 1328, 1368, 1201, 1180, 1114, 1087, 1067, $1026,994,971,952,935,865,813 \mathrm{~cm}^{-1}$.

\section{4-Benzoyl-5-methyl-1H-imidazol-2(3H)-one (7b)}

(E)-3-(dimethylamino)-1-phenylbut-2-en-1-one ${ }^{29}$ (2b) (3.78 g, $20 \mathrm{mmol}$ ) was disolved in $\mathrm{MeOH}$ $(50 \mathrm{~mL}) \mathrm{NH}_{4} \mathrm{OAc}$ was added $(15.4 \mathrm{~g}, 200 \mathrm{mmol})$ and the reaction mixture was stirred for $3 \mathrm{~h}$ at room temperature. Volatile components were evaporated and the product, (Z)-3-amino-1phenylbut-2-en-1-one, ${ }^{30}$ was isolated by column chromatography (EtOAc/petroleum ether = 1:1) and used directly in the next step. EtOH $(50 \mathrm{~mL})$ and DEAD was added $(2.98 \mathrm{~mL}, 19.0 \mathrm{mmol})$ and the reaction mixture was stirred $14 \mathrm{~h}$ at room temperature when $\mathrm{NaOH}(1.90 \mathrm{~g}, 47.5 \mathrm{mmol})$ was added. The reaction mixture was stirred further for $24 \mathrm{~h}$. Precipitated product was used directly in the next step. Ethyl (5-benzoyl-4-methyl-2-oxo-2,3-dihydro-1H-imidazol-1yl)carbamate (5b) $(662 \mathrm{mg}, 2.3 \mathrm{mmol})$ was dissolved in the mixture of $\mathrm{H}_{2} \mathrm{O} / \mathrm{THF}=1: 1(20 \mathrm{~mL})$ and $\mathrm{KOH}$ was added to solution $(560 \mathrm{mg}, 10 \mathrm{mmol})$. The reaction mixture was refluxed for $24 \mathrm{~h}$, volatile components were evaporated and intermediate 1-amino-5-benzoyl-4-methyl-1Himidazol-2(3H)-one (6b) was isolated by column chromatography $\left(\mathrm{CHCl}_{3} / \mathrm{MeOH}=7: 1\right)$. Intermediate $6 \mathbf{a}$ was suspended in $\mathrm{H}_{2} \mathrm{O}(10 \mathrm{~mL})$ and $\mathrm{HCl}_{(\mathrm{aq})}(2 \mathrm{~mL})$ was added to a suspension. Reaction mixture was chilled to $0{ }^{\circ} \mathrm{C}$ and $1.5 \mathrm{M}$ solution of $\mathrm{NaNO}_{2(\mathrm{aq})}(8 \mathrm{~mL})$ was added during 
30 minutes. Reaction mixture was than stirred for $2 \mathrm{~h}$ at room temperature. Volatile components were evaporated and the product was isolated using Soxhlet's extraction (EtOAc). Yield: $353 \mathrm{mg}$ (76\%), yellow solid, mp 243-246 ${ }^{\circ} \mathrm{C}\left(\mathrm{mp}^{17} 253-255{ }^{\circ} \mathrm{C}\right) .{ }^{1} \mathrm{H}$ NMR (DMSO- $\left.d_{6}, 300 \mathrm{MHz}\right): \delta 1.81$ $\left(3 \mathrm{H}, \mathrm{s}, \mathrm{CH}_{3}\right) ; 7.45-7.52(2 \mathrm{H}, \mathrm{m}, \mathrm{Ph}) ; 7.54-7.61(3 \mathrm{H}, \mathrm{m}, \mathrm{Ph}) ; 10.27(1 \mathrm{H}$, br s, NH); $10.84(1 \mathrm{H}, \mathrm{br}$ $\mathrm{s}, \mathrm{N} H)$.

\section{4-(4-Chlorobenzoyl)-5-methyl-1H-imidazol-2(3H)-one (7c)}

Ethyl (5-(4-chlorobenzoyl)-4-methyl-2-oxo-2,3-dihydro-1H-imidazol-1-yl)carbamate (5c) (1.62 $\mathrm{g}, 5 \mathrm{mmol})$ was dissolved in $\mathrm{EtOH}(20 \mathrm{~mL}) \mathrm{KOH}$ was added $(3.00 \mathrm{~g}, 53.5 \mathrm{mmol})$ and the reaction mixture was refluxed for $15 \mathrm{~h}$. Volatile components were evaporated, solid residue dissolved in $\mathrm{H}_{2} \mathrm{O}(5 \mathrm{~mL})$ and concentrated $\mathrm{HCl}_{(\mathrm{aq})}(3 \mathrm{~mL})$ was added. Reaction mixture was than chilled to $0{ }^{\circ} \mathrm{C}$ and $6 \mathrm{M} \mathrm{NaNO}_{2 \text { (aq) }}(5 \mathrm{~mL})$ was slowly added during $20 \mathrm{~min}$. When all $\mathrm{NaNO}_{2 \text { (aq) }}$ was added the reaction mixture was stirred further for $20 \mathrm{~min}$ at room temperature. White solid precipitated which was filtered and washed with $\mathrm{Et}_{2} \mathrm{O}(3 \times 5 \mathrm{~mL})$. Recrystallization from EtOH. Yield: $944 \mathrm{mg}\left(80 \%\right.$ ), white solid, mp 284.3-286.9 ${ }^{\circ} \mathrm{C}\left(\mathrm{mp}^{17} 291-293{ }^{\circ} \mathrm{C}\right) .{ }^{1} \mathrm{H}$ NMR (DMSO- $d_{6}$, $500 \mathrm{MHz}): \delta 1.86\left(3 \mathrm{H}, \mathrm{s}, \mathrm{CH}_{3}\right) ; 7.55(2 \mathrm{H}, \mathrm{d}, J=8.5 \mathrm{~Hz}, \mathrm{Ph}) ; 7.63(2 \mathrm{H}, \mathrm{d}, J=8.5 \mathrm{~Hz}, \mathrm{Ph}) ; 10.34$ $(1 \mathrm{H}$, br s, $\mathrm{N} H) ; 10.94(1 \mathrm{H}$, br s, $\mathrm{N} H) .{ }^{13} \mathrm{C} \mathrm{NMR}$ (DMSO- $\left.d_{6}, 126 \mathrm{MHz}\right): \delta 12.1,118.9,128.6$, 130.0, 132.7, 136.2, 137.9, 152.7, 182.2. $\left(\mathrm{C}_{11} \mathrm{H}_{9} \mathrm{~N}_{2} \mathrm{O}_{2} \mathrm{Cl}\right.$ calculated: $\mathrm{C}, 55.83 ; \mathrm{H}, 3.83 ; \mathrm{N}, 11.84$. found $\mathrm{C}$, 55.63; H, 3.62; N, 11.70); EI-HRMS: $m / z=237.0430\left(\mathrm{MH}^{+}\right) ; \mathrm{C}_{11} \mathrm{H}_{10} \mathrm{~N}_{2} \mathrm{O}_{2} \mathrm{Cl}$ calculated: $\mathrm{m} / \mathrm{z}=237.0425\left(\mathrm{MH}^{+}\right) ; v_{\max }(\mathrm{KBr}) 2967,1661,1614,1592,1488,1437,1437,1394,1373,1316$, $1267,1182,1127,1086,1013,949,936,836 \mathrm{~cm}^{-1}$.

\section{4-(4-Bromobenzoyl)-5-methyl-1H-imidazol-2(3H)-one (7e)}

Ethyl (5-(4-bromobenzoyl)-4-methyl-2-oxo-2,3-dihydro-1H-imidazol-1-yl)carbamate (5e) (421.5 $\mathrm{mg}, 1.5 \mathrm{mmol})$ was dissolver in $\mathrm{EtOH}(5 \mathrm{~mL}) \mathrm{KOH}$ was added $(1.00 \mathrm{~g}, 17.85 \mathrm{mmol})$ and reaction mixture refluxed for $12 \mathrm{~h}$. Volatile components were evaporated the solid residue dissolved in $\mathrm{H}_{2} \mathrm{O}(5 \mathrm{~mL})$ and concentrated solution of $\mathrm{HCl}_{(\mathrm{aq})}(2 \mathrm{~mL})$ was added. Reaction mixture was than chilled to $0{ }^{\circ} \mathrm{C}$ and $6 \mathrm{M} \mathrm{NaNO}_{2(\mathrm{aq})}(5 \mathrm{~mL})$ was slowly added during $20 \mathrm{~min}$. When all $\mathrm{NaNO}_{2(\mathrm{aq})}$ was added the reaction mixture was stirred further for $20 \mathrm{~min}$ at room temperature. White solid precipitated which was filtered and washed with $\mathrm{Et}_{2} \mathrm{O}(3 \times 5 \mathrm{~mL})$. Recrystallization from $\mathrm{H}_{2} \mathrm{O}$. Yield: $279 \mathrm{mg}(87 \%)$, white solid, mp 246.7-249.9 ${ }^{\circ} \mathrm{C} .{ }^{1} \mathrm{H}$ NMR (DMSO- $\left.d_{6}, 300 \mathrm{MHz}\right): \delta 1.86\left(3 \mathrm{H}, \mathrm{s}, \mathrm{CH}_{3}\right) ; 7.54(2 \mathrm{H}, \mathrm{d}, J=8.4 \mathrm{~Hz}, \mathrm{Ph}) ; 7.69(2 \mathrm{H}, \mathrm{d}, J=8.4 \mathrm{~Hz}$, $\mathrm{Ph}) ; 10.30\left(1 \mathrm{H}\right.$, br s, NH); 10.91 (1H, br s, NH). ${ }^{13} \mathrm{C}$ NMR (DMSO- $\left.d_{6}, 75.5 \mathrm{MHz}\right): \delta 12.1,118.8$, 125.0, 130.1, 131.4, 132.6, 138.2, 152.7, 182.3. $\left(\mathrm{C}_{11} \mathrm{H}_{9} \mathrm{~N}_{2} \mathrm{O}_{2} \mathrm{Brx} 1 / 4 \mathrm{H}_{2} \mathrm{O}\right.$ calculated: $\mathrm{C}, 46.26 ; \mathrm{H}$, 3.35; N, 9.81. found $\mathrm{C}, 4.32 ; \mathrm{H}, 3.06 ; \mathrm{N}, 9.75) ; \mathrm{EI}-\mathrm{HRMS}: \mathrm{m} / \mathrm{z}=280.9916\left(\mathrm{MH}^{+}\right)$; $\mathrm{C}_{11} \mathrm{H}_{10} \mathrm{~N}_{2} \mathrm{O}_{2} \mathrm{Br}$ calculated: $\mathrm{m} / \mathrm{z}=280.9920\left(\mathrm{MH}^{+}\right) ; v_{\max }(\mathrm{KBr}) 3413,1702,1617,1465,1438$, $1393,1329,1269,1170,1124,1067,1044,1011,936,836 \mathrm{~cm}^{-1}$. 


\section{X-ray structure analysis for compound $2 \mathrm{~d}$}

The reflection data were collected on a Nonius Kappa CCD diffractometer using monochromated Mo Ka radiation at room temperature by using Nonius Collect software. ${ }^{32}$ Data reduction and integration were performed with the software package DENZO-SMN. ${ }^{33}$ The coordinates of all of the nonhydrogen atoms were found via direct methods using the SIR97 structure solution program. $^{34}$ A full-matrix least-squares refinement on $F^{2}$ magnitudes with anisotropic displacement parameters for all non-hydrogen atoms using SHELXL-97 was employed. ${ }^{35}$ All H atoms were initially located in difference Fourier maps and subsequently treated as riding atoms in geometrically idealized positions with bond lengths $\mathrm{C}-\mathrm{H} 0.96 \AA$ for methyl and $0.93 \AA$ for aromatic hydrogens with $\operatorname{Uiso}(\mathrm{H})=1.5 \mathrm{Ueq}(\mathrm{C}$, methyl) and $\operatorname{Uiso}(\mathrm{H})=1.2 \mathrm{Ueq}(\mathrm{C}$, aromatic $)$, respectively. Figures depicting the structures were prepared by ORTEP3. ${ }^{36}$ CCDC-939062 contains the supplementary crystallographic data for structure $\mathbf{2 d}$. These data can be obtained free of charge from The Cambridge Crystallographic Data Centre via www.ccdc.cam.ac.uk/data_request/cif.

\section{Acknowledgements}

Financial support from the Slovenian Research Agency through grants P0-0502-0103, P1-0179 and J1-6689-0103-04 and grant Packet X-2000 and PS-511-102 for the purchase of Kappa CCD Nonius diffractometer are gratefully acknowledged. We also thank the Krka d.d. (Novo mesto, Slovenia) and Lek d.d., a Sandoz Company (Ljubljana, Slovenia) for financial support.

\section{References}

1. Chebanov, A. V.; Desenko, S. M.; Gurley, T. W. Azaheterocycles Based on $\alpha, \beta$-Unsaturated Carbonyl, Springer, Berlin 2008.

2. Jin, Z. Nat. Prod. Rep. 2009, 26, 382-445. http://dx.doi.org/10.1039/b718045b

3. De Luca, L. Curr. Med. Chem. 2006, 13, 1-23.

4. Suijkerbuijk, B. M. J. M.; Niculescu-Duvaz, I.; Gaulon, C.; Dijkstra, H. P.; Niculescu-Duvaz, D.; Menard, D.; Zambon, A.; Nourry, A.; Davies, L.; Manne, H. A.; Friedlos, F.; Ogilvie, L. M.; Hedley, D.; Lopes, F.; Preece, N. P. U.; Moreno-Farre, J.; Raynaud, F. I.; Kirk, R.; Whittaker, S.; Marais, R.; Springer, C. J. J. Med. Chem. 2010, 53, 2741-2756. http://dx.doi.org/10.1021/jm900607f

5. Finke, P. E.; Meurer, L. C.; Levorse, D. A.; Mills, S. G.; MacCoss, M.; Sadowski, S.; Cascieri, M. A.; Tsao, K.-L.; Chicchi, G. G.; Metzger, J. M.; MacIntyre, D.E. Bioorg. Med. Chem. Lett. 2006, 16, 4497-4503. http://dx.doi.org/10.1016/j.bmcl.2006.06.035 
6. Carling, R. W.; Moore, K. W.; Moyes, C. R.; Jones, E. A.; Bonner, K.; Emms, F.; Marwood, R.; Patel, Sh.; Patel, S.; Fletcher, A. E.; Beer, M.; Sohal, b.; Pike, A.; Leeson, P. D. J. Med. Chem. 1999, 42, 2706-2715. http://dx.doi.org/10.1021/jm991029k

7. De Clercq, J. P. Chem. Rev.1997, 97, 1755-1792. http://dx.doi.org/10.1021/cr9704671

8. Sosa, A. C. B.; Yakushijin, K.; Horne, D. A. Org. Lett. 2000, 2, 3443-3444. http://dx.doi.org/10.1021/ol000233v

9. Sosa, A. C. B.; Yakushijin, K.; Horne, D. A. J. Org. Chem. 2002, 67, 4498-4500. http://dx.doi.org/10.1021/jo020063v

10. Dransfield, P. J.; Dilley, A. S.; Wang, S.; Romo, D. Tetrahedron 2006, 62, 5223-5247. http://dx.doi.org/10.1016/j.tet.2005.12.068

11. Selič, L.; Jakše, R.; Lampič, K.; Golič, L.; Golič-Grdadolnik, S.; Stanovnik, B. Helv. Chim. Acta 2000, 83, 2802-2811. http://dx.doi.org/10.1002/1522-2675(20001004)83:10<2802::AID-HLCA2802>3.0.CO;2-9

12. Koswatta, P. B.; Sivappa, R.; Dias, H. V. R.; Lovely, C. J. Synthesis 2009, 2970-2982. http://dx.doi.org/10.1055/s-0029-1216929

13. Grimmet. M. R. Product Class 3: Imidazoles in Science of Synthesis, Georg Thieme Verlag, Stuttgart 2006; Vol 12, pp. 325-528.

14. Dabdab, M; Renault, S.; Eid, S.; Lozach, O.; Meijer, L.; Carreaux, F.; Bazureau, J. P. Heterocycles 2009, 78, 1191-1203. http://dx.doi.org/10.3987/COM-08-11594

15. Evano, G.; Blanchard, N.; Toumi, M. Chem. Rev. 2008, 108, 3054-3131. http://dx.doi.org/10.1021/cr8002505

16. Gong, X.; Yang, H.; Liu, H.; Jiang, Y.; Zhao, Y.; Fu, H. Org. Lett. 2010, 12, 3128-3131. http://dx.doi.org/10.1021/ol1008813

17. Schnettler, R.; Dage, R. C.; Grisar, J. M. J. Med. Chem. 1982, 25, 1477-1481. http://dx.doi.org/10.1021/jm00354a017

18. Innes, P. A.; Fraser, R. S.; Booker, P. D.; , Allsop, E.; Kirton, C.; Lockie, J.; Franks, R. Br. J. Anaesth. 1994, 72, 77-81.

http://dx.doi.org/10.1093/bja/72.1.77

19. Booker, P. D.; Gibbons, S.; Stewart, J. I. M.; Selby, A.; Wilson-Smith, E.; Pozzi, M. Br. J. Anaesth. 2000, 85, 205-210.

http://dx.doi.org/10.1093/bja/85.2.205

20. Ruef, P.; Craciun, E.; Altfelder, F.; Simon, C.; Frommhold, D.; Koch, L.; Poeschel, J. Clin. Hemorheol. Micro. 2010, 45, 301-310.

21. Stanovnik, B.; Svete, J. Chem. Rev., 2004, 104, 2433-2480.

http://dx.doi.org/10.1021/cr020093y

22. Čebašek, P.; Wagger, J.; Bevk, D.; Jakše, R.; Svete, J.; Stanovnik, B. J. Comb. Chem. 2004, 6, 356-362. 
http://dx.doi.org/10.1021/cc034066c

23. Grošelj. U.; Rečnik, S.; Svete, J.; Meden, A.; Stanovnik, B. Tetrahedron: Asymmetry 2002, $13,821-833$.

http://dx.doi.org/10.1016/S0957-4166(02)00208-2

24. Selič, L.; Stanovnik, B. Tetrahedron 2001, 57, 3159-3164.

http://dx.doi.org/10.1016/S0040-4020(01)00174-0

25. Časar, Z.; Bevk, D.; Svete, J.; Stanovnik, B. Tetrahedron 2005, 61, 7508-7519. http://dx.doi.org/10.1016/j.tet.2005.05.075

26. Wagger, J; Grošelj, U.; Meden, A.; Svete, J.; Stanovnik, B. Tetrahedron 2008, 64, 2801 2815. http://dx.doi.org/10.1016/j.tet.2008.01.045

27. Wagger, J.; Svete, J.; Stanovnik, B. Synthesis 2008, 1436-1442.

http://dx.doi.org/10.1055/s-2008-1072515

28. Bezenšek, J.; Grošelj, U.; Stare, K.; Svete, J.; Stanovnik B. Tetrahedron 2012, 68, 516-522. http://dx.doi.org/10.1016/j.tet.2011.11.013

29. Mukhanova, T. I.; Granik, V. G.; Denosov, A. V.; Trubitsina, T. K.; Shvarts, G. Y.; Mashkovsky, M. D. Khim. Farm. Zh. 1994, 28, 23-26.

30. Singh, B.; Lesher, G. V. J. Heterocycl. Chem. 1990, 27, 2085-2091. http://dx.doi.org/10.1002/jhet.5570270743

31. Lin Y.-I.; Lang, S. A. Jr. J. Org. Chem., 1980, 45, 4857-4860.

http://dx.doi.org/10.1021/jo01312a011

32. Collect Software; Nonius, BV: Delft, The Netherlands, 2000.

33. Otwinowski, Z.; Minor, W. Methods Enzymol. 1997, 276, 307-326. http://dx.doi.org/10.1016/S0076-6879(97)76066-X

34. Altomare, A.; Burla, M. C.; Camalli, M.; Cascarano, G. L.; Giacovazzo, C.; Guagliardi, A.; Moliterni, A. G. G.; Polidori, G.; Spagna, R. J. Appl. Crystallogr. 1999, 32, 115-119. http://dx.doi:10.1107/S0021889898007717

35. Sheldrick, G. M. Acta Crystallogr. 2008, A64, 112-122.

http://dx.doi.org/10.1107/S0108767307043930

36. Farrugia, L. J. J. Appl. Crystallogr. 1997, 30, 565.

http://dx.doi:10.1107/S0021889897003117 